\title{
Comprehensive Study on Shape Shifting Behaviors of Thermally Activated Hinges in FDM-based 4D Printing
}

Iman Salimi Nezhad

Tarbiat Modares University Faculty of Engineering https://orcid.org/0000-0002-9223-2268

Mohammad Golzar ( $\sim$ m.golzar@modares.ac.ir)

Tarbiat Modares University Faculty of Engineering

Amirhossein Behravesh

Tarbiat Modares University Faculty of Engineering

Shahabaddin Zare

Tarbiat Modares University Faculty of Engineering

\section{Research Article}

Keywords: 4D printing, FDM, Shape-Shifting, PLA, Constitutive model

Posted Date: August 24th, 2021

DOI: https://doi.org/10.21203/rs.3.rs-791407/v1

License: (c) (i) This work is licensed under a Creative Commons Attribution 4.0 International License.

Read Full License 


\title{
Comprehensive study on shape shifting behaviors of thermally activated hinges in FDM-based 4D printing
}

\author{
Iman Salimi Nezhad $^{1}$, Mohammad Golzar $^{1 *}$, Amirhossein Behravesh $^{1}$, Shabbadin $_{\text {Zare }}^{1}$
}

${ }^{1}$ Additive Manufacturing Laboratory, Faculty of Mechanical Engineering, Tarbiat Modares University (TMU),

Jalal AleAhmad, Tehran 14115-111, Iran

* Corresponding author:

E-mail:m.golzar@modares.ac.ir

Office: $+98-21-82884320$

Fax: $+98-21-82884320$ 


\begin{abstract}
4D printing of shape shifting structures, aka "hinges", has raised a new standard in many fields. By using these hinges in certain parts of a 3D printed structures, a pre designed complex 3D shape with potential multifunctional application can be achieved from flat structure. This paper proposes a comprehensive semi-empirical model to predict the final shape shifting behavior and magnitude of the hinges printed by FDM process. First, all FDM main parameters are selected and reduced by design of experiment to printing speed, lamina thickness, nozzle temperature as well as printing pattern. In order to develop the model, a time-dependent constitutive model with these four process parameters were extracted for strain of an SMP homogeneous single layer structure using a fractional Zener model accompanied with Multiple Linear Regression (MLR) technique. Thereafter, the mathematical relations for shape shifting behavior of bilayer 4D printed structures were developed for beam bending and twisting by modifying Timoshenko's constitutive equations. A comprehensive shape-shifting model was established including 3D printing parameters, angles, thickness ratios, activation time and temperature which was compared to the experimental data and results predicted both shape shifting behavior and magnitude of the hinges with good agreement. In addition, a novel flowchart was suggested to design and achieve the desired shape shifting behaviors. The proposed model and flowchart are novel tools to design 4D structures through desired shape-shifting of the hinges.
\end{abstract}

Keywords: 4D printing, FDM, Shape-Shifting, PLA, Constitutive model. 


\section{INTRODUCTION}

The term “4D printing” was introduced by Tibbits in 2013 [1] and refers to the 3D printing processes in which the resulting object could changes its shape with time (i.e., the $4^{\text {th }}$ dimension) [1-3]. In most cases, the working basis of 4D printing is shape-shifting in synthetic materials, which is achieved either by a physical effect such as residual stresses or through the energy released from an active or smart material [4]. Most of the 3D printing techniques, such as material jetting [5-8], DW [9], SLA [10]\& [11], and customized 3D printers [12,13] are suitable for use in 4D printing processes, but not all can be used equally well as they usually require specialized equipment that put them out of the reach of most research labs; Moreover, the materials used in these processes tend to be expensive and proprietary. Recently, material extrusion technique (FDM), which is one of the most commonly available and inexpensive 3D printing processes that uses affordable off-the-shelf materials, is receiving increasing attention [14-22] as a good replacement for the abovementioned processes.

There are two approaches to 4D printing using an FDM 3D printer. The first approach is an approach that requires post-processing after 3D printing, i.e., manually deforming the printed object into a temporary shape after 3D printing, which complicates the manufacturing process. Unlike the first approach, the second approach uses only a 3D printer, without the need for post-processing to manually change the shape of the printed structure [23]. It also eliminates the need to print supports and reduces the printing time compared with direct printing of the targeted shapes [24]. In this approach a flat bilayer structure composed of two layers with different strains while exposed to an external stimulus is printed. In recent years, 4D printing of these shape-shifting flat structures aka active hinges has raised a new standard in many fields such as bio-technology, electronics, aerospace, etc. [25]. These hinges can be used in certain parts of an structure, enabling the user to bend or twist the structure where needed and produce a complex shape out of a simple structure [26]. Making the flat structure, aside from the simplicity, will give the user access to the surface of the structure, which can be used for many purposes including drug delivery devices [27]. Applying active hinges in certain parts of a complex structures enables the structure to be more compact and portable. On the other hand, controlled and precise self-shape-shifting of these hinges eliminates the need for human intervention. Thus, there are a lot of potential and actual applications for them specially for remote and space-limited environments like spaceships. Figure 1 shows one of these potential applications. A device that carries two planar antennas or two solar panels which uses active hinges. Each side of the device have one antenna/panel. As it can be seen in Figure 1, the device is flat, compact and portable before activation. Right antenna/panel is attached to two hinges that are expected to only bend 60 degrees and left antenna/panel is attached to a hinge that is expected to bend 60 degrees and twist 35 degrees. We used our model 
to set the process parameters so that the desired conditions are satisfied. Printed specimen is also shown in Figure 1.

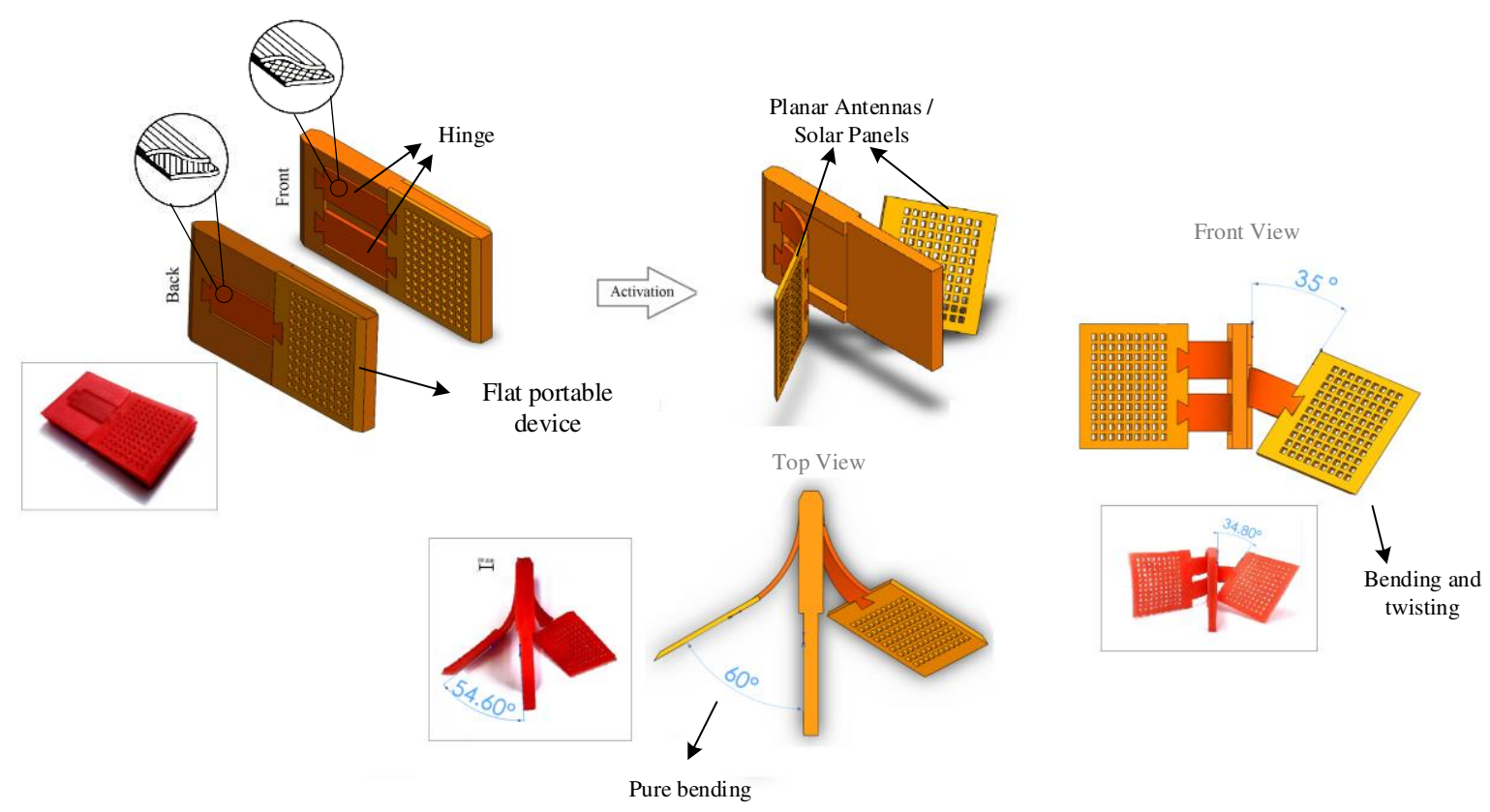

Figure 1. a potential application of designing the $4 D$ printed hinges in structures

In order to understand and predict the shape shifting behavior of 4D printed structures, researchers have proposed various models and simulations. Zhang et al [28] Studied a simple structure of long PLA strip which printed by FDM process and proposed a viscoelastic model using Zener's first mode model to calculate the internal strain stored in 3D printed structures. In their next work [29], they introduced an effective thermal expansion coefficient for their material and modeled the final bending angle of the strips. Liu et al [30] studied the effect of the printing pattern as well as the printing angles of the layers on the mechanical properties of anisotropic FDMbased 4D printed structures. They used the classical lamination theory (CLT) to calculate the elastic modulus of the structure. They showed that using CLT, the equivalent elastic modulus for different patterns can be calculated with good accuracy. Liu et al [31] presented a model for predicting the deformation of 4D printed bilayer structures .They defined a new hyperplastic energy density function to calculate the energy of a bilayer structure during bending. By minimizing the energy of the bilayer structure, a flexural deformation model was created by considering the thickness ratio. Hernandez et al [32] did a parametric study to interpret the effects of different manufacturing parameters on the mechanical performance of ABS specimens and proposed an experimentalmodeling to predict mechanical properties of the structures printed via FDM process. Zeng et al [33] formulated a 4D printing mathematical model considering printing process parameters to predict the curvature of self-folding hinges. Bodaghi et al [34] presented a finite element method (FEM) in Abaqus to simulate the thermo-mechanical 
behavior of the 4D printed structures. To date, most mathematical and numerical studies on FDM-based 4D printing have focused on predicting the mechanical and thermo-mechanical behavior of these structures through physical and material parameters. There are only handful of studies concerning the shape-shifting predictions through 3D printing process parameters, and they only model the bending behaviors.

here in this study, we provide a semi-empirical constitutive model to predict the time dependent shape-shifting magnitude and behavior of the hinges including bending, twisting and a combination of both, through printing parameters namely printing speed, lamina thickness and nozzle temperature which were selected among all important process parameters using $\mathrm{S} / \mathrm{N}$ ratio analysis. In addition, the model is compatible with angle laminae. Thus this proposed model will grant the most comprehensive control over 4D printed structures through active hinges.

\section{Methods}

\subsection{Working principle}

4D printed structures require an interaction mechanism in order to properly respond to the applied stimulus. Different interaction mechanisms have been proposed in the literature [25], of which a specific one called "constrained-thermo-mechanics" happens in FDM process. Figure 2 shows a schematic of FDM process where SMP filament is extruded through the hot end nozzle. First, the SMP is heated to $T_{H}$ that is above its glass transition temperature $\left(T_{g}\right)$ through the hot end nozzle and is extruded on the bed at this temperature which causes the orientation of the polymer chains in nozzle movement direction (print pattern). while nozzle moving forward, the printed SMP is cooled to $T_{L}$ that is below its $T_{g}$. When cooled to $T_{L}$, the orientation imposed onto the SMP will be maintained; this is simply due to the freezing of the polymer chains that locks in the oriented chain conformation, enabling the storage of entropic energy which produces a stored strain in the SMP. when it is heated above the $T_{g}$ again in the absence of external stress, the SMP will release the strain and recover to its permanent shape. heating activates the molecular mobility, which releases the entropic energy, thus driving the molecular chains back into its highest entropic state corresponding to the permanent shape [35]. 


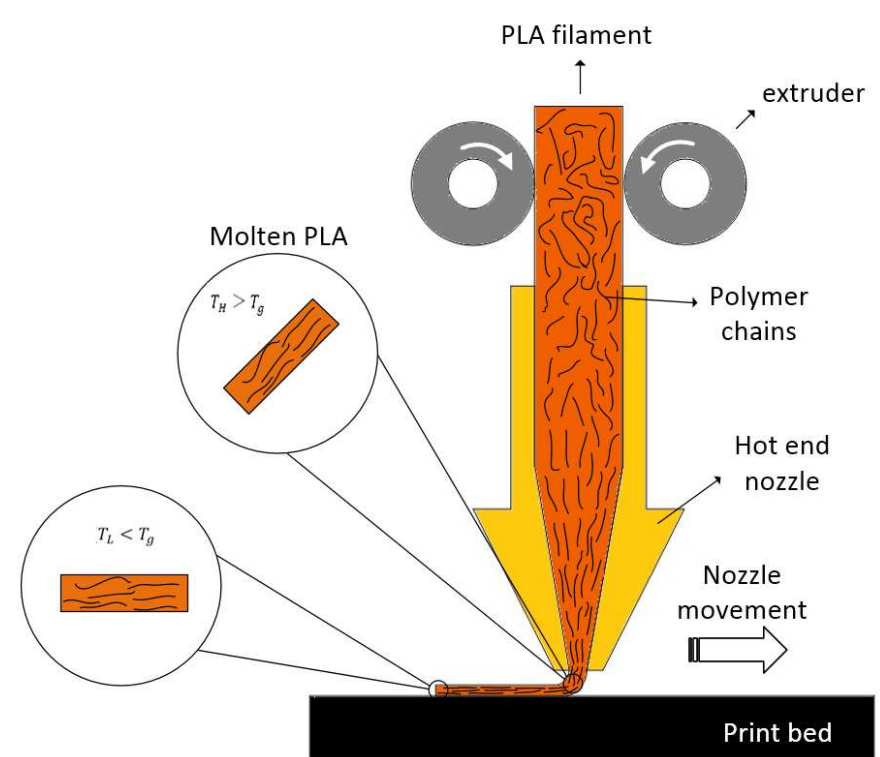

Figure 2. schematic illustration of the constrained thermomechanical mechanisms in $4 D$ printing.

Now to extract the out of plane shape shifting behaviors such as bending and twisting from this shape memory strains, the concept of bilayer structures is being used. Figure 3 shows the concept of a bilayer strip (hinge) in FDM 3D printing process, with each layer having a different printing pattern and/or process parameters. As it can be seen in Figure 3 (right) each layer consists of an arbitrary number of laminae and each lamina consists of an arbitrary number of rasters. This difference in printing pattern and/or process parameters leads to different stored strain in each layer which according to Timoshenko's bilayer beam theory [36] will cause out of plane shape shifting behaviors (Figure 3, left). By changing the strain difference of two layers (longitudinal, transverse or shear strains), the shape-shifting behavior and its magnitude can be controlled. Figure 4 shows six apparently similar printed samples with different shape-shifting behaviors indicating the importance of programming these strains through printing parameters and printing patterns.
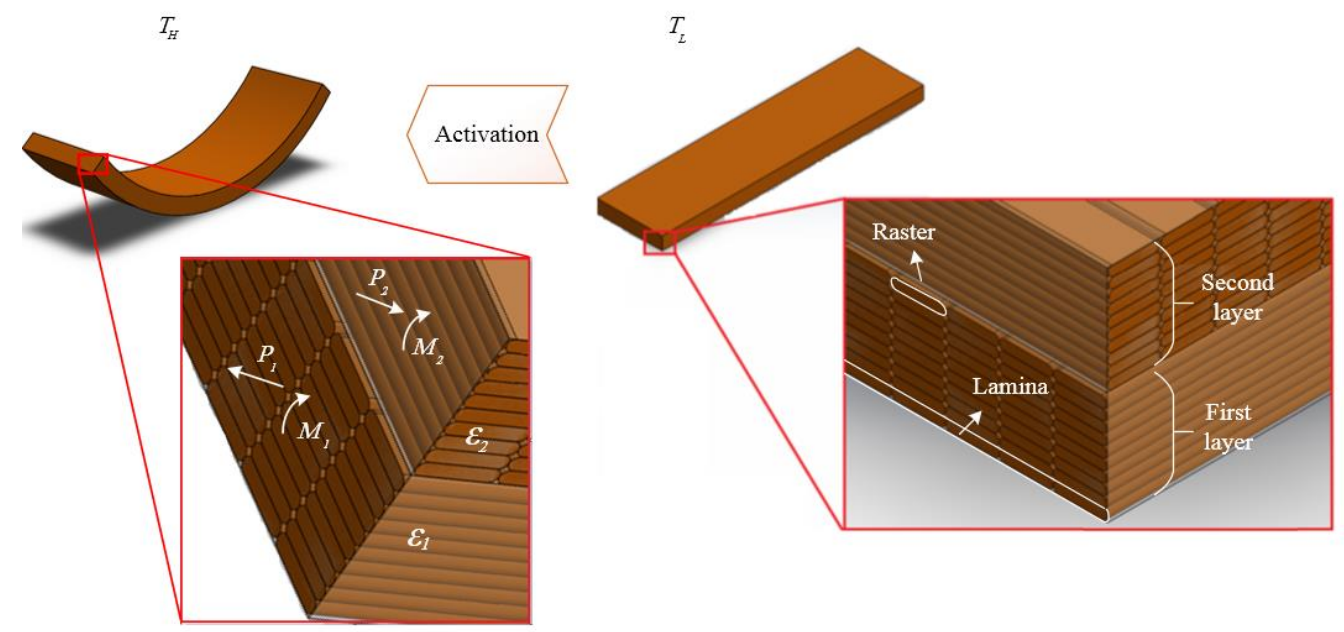

Figure 3. (right) As-printed bilayer strip and its components, (left) when the temperature rise above Tg the strip shows out of plane shape-shifting behaviors duo to the strain difference of two layers 


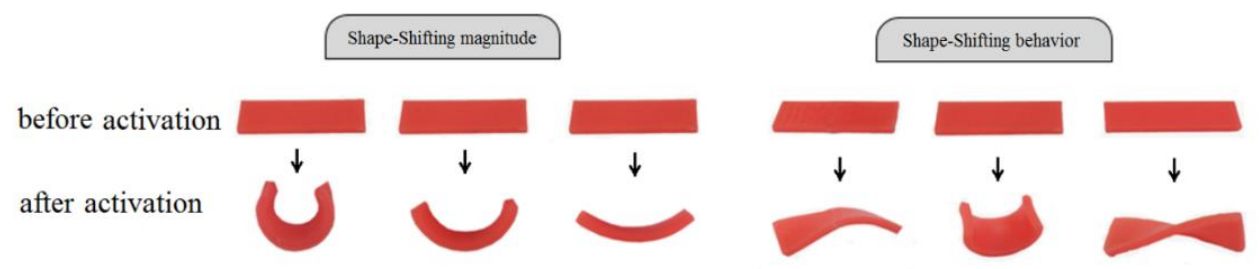

Figure 4. By manipulating the longitudinal, transverse and shear strain of each layer, the shape-shifting behavior and magnitude can be controlled.

\subsection{Materials and fabrication process}

PLA (Poly-Lactic Acid) filament with diameter of $1.75 \mathrm{~mm}$ was used in all experiments. An FDM printer (Sizan3, Sizan Pardasezh Kavir, Iran) with a nozzle diameter of $0.5 \mathrm{~mm}$ was used for printing all specimens at room temperature. Activation time and temperature were set to 30 seconds and 90 Celsius degrees respectively. specimens were heated using a laboratory hot water bath and digital image correlation (DIC) technique used to measure the strains curvatures and twists which were performed in triplicates. DSC measurements were performed using a NETZSCH DSC instrument (200F3) to characterize the thermal properties of the PLA filament. Approximately $9 \mathrm{mg}$ of the filament was heated from $0{ }^{\circ} \mathrm{C}$ to $250{ }^{\circ} \mathrm{C}$ at a rate of $10^{\circ} \mathrm{C} / \mathrm{min}$. the glass transition temperature $\left(T_{g}\right)$, and melting temperature $\left(T_{m}\right)$ were obtained about $65{ }^{\circ} \mathrm{C}$ and $165^{\circ} \mathrm{C}$ respectively.

Figure 5 shows an FDM 3D printer printing samples with different printing patterns. According to Figure 5, [ $\theta]$ print pattern means that all laminae are printed at an angle of $\theta(-90 \leq \theta \leq 90)$ degrees to the horizon. $[ \pm \theta]$ Print pattern means even laminae are printed with a positive angle $\theta$ and even laminae are printed with a negative angle $\theta$. The table in Figure 5 shows printing conditions used to print samples for this study.

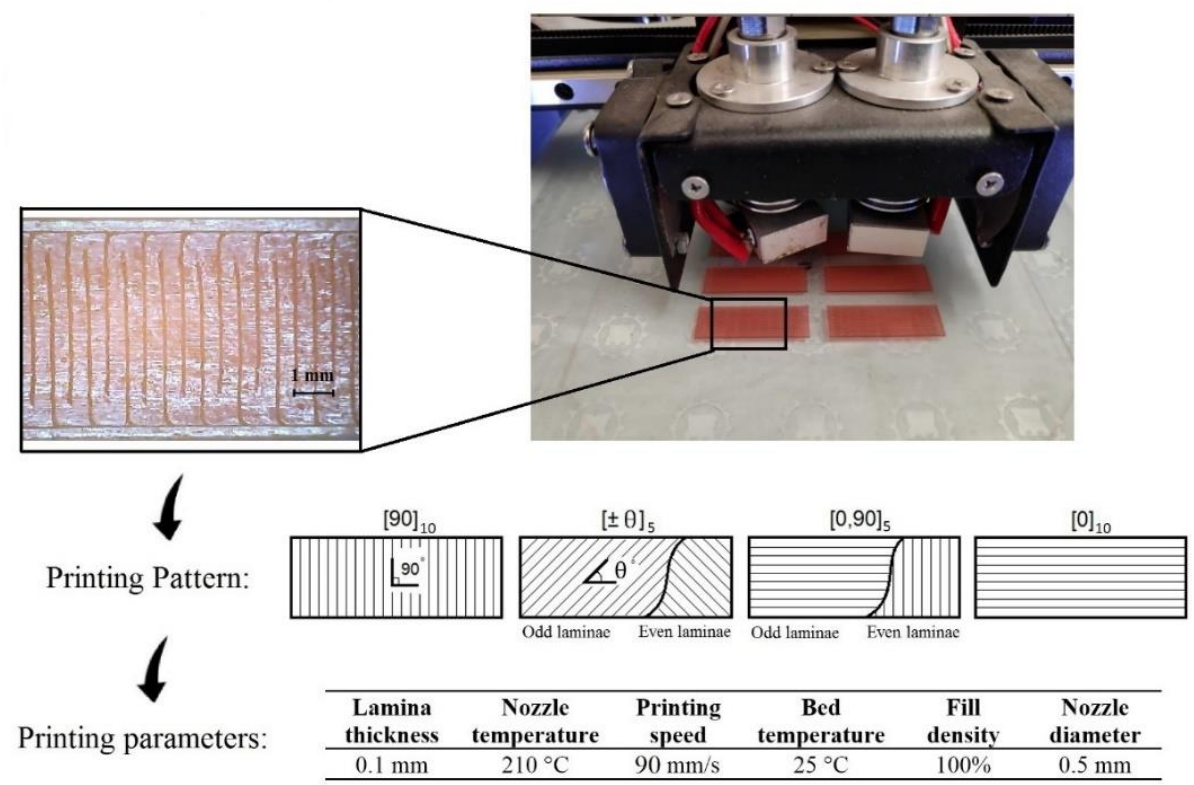

Figure 5. printing patterns and conditions used to print samples with an FDM 3D printer 


\subsection{Shape recovery ratio test}

Four specimens $(40 \times 10 \times 2 \mathrm{~mm})$ were printed according to the printing parameters given in Figure 5 . A tensile strain of 10 percent were applied to Each specimen using SANTAM STM-20 tensile tester following the thermomechanical mechanism: the specimens were first heated to their $T_{g}\left(65^{\circ} \mathrm{C}\right)$ in a hot water bath installed on the tensile tester, then were loaded to the predetermined strain while the temperature was held at $65^{\circ} \mathrm{C}$. the specimens then were cooled down to room temperature without removing the external stress so as to maintain the strain. For the final step, each specimen were reheated to a different temperature $\left(65-95^{\circ} \mathrm{C}\right)$. A Dino-Light AM3713BT digital microscope were used to capture the shape recovery in time.

\subsection{Design of experiments}

In this study, all of the main printing parameters that may affect the amount of strain which is the cause of shapeshifting behavior in the $4 \mathrm{D}$ printing process, were selected. Using the Taguchi method and signal to noise $(\mathrm{S} / \mathrm{N})$ ratio analysis the parameters with significant effect on the strain were determined. Table 1 shows the parameters and their levels (only two levels are required for screening). According to the number of parameters and their levels, L8 Taguchi Orthogonal Array design [37] were chosen to implement $\mathrm{S} / \mathrm{N}$ ratio analysis. Table 2 shows the L8 design for printing parameters.

Table 1. main printing parameters and their levels for screening

\begin{tabular}{|c|c|c|c|c|c|c|c|c|}
\hline & \multicolumn{8}{|c|}{ Factors (parameters) } \\
\hline & & $\begin{array}{l}\text { Printing Speed } \\
\qquad(\mathrm{mm} / \mathrm{s})\end{array}$ & $\begin{array}{c}\text { Nozzle } \\
\text { Temperature }\left({ }^{\circ} \mathrm{C}\right)\end{array}$ & $\begin{array}{c}\text { Bed Temperature } \\
\left({ }^{\circ} \mathrm{C}\right)\end{array}$ & Fill Percentage & $\begin{array}{c}\text { Nozzle } \\
\text { Diameter } \\
(\mathrm{mm})\end{array}$ & $\begin{array}{c}\text { Lamina } \\
\text { Thickness (mm) }\end{array}$ & $\begin{array}{l}\text { Extrusion } \\
\text { Multiplier }\end{array}$ \\
\hline \multirow{2}{*}{ levels } & 1 & 30 & 180 & 25 & 75 & 0.2 & 0.05 & 0.8 \\
\hline & 2 & 120 & 230 & 50 & 100 & 0.5 & 0.2 & 1.2 \\
\hline
\end{tabular}

Table 2. main printing parameters and their levels for screening according to L8 Taguchi Orthogonal Array

\begin{tabular}{cccccccc}
\hline \multirow{2}{*}{ Exp. } & \multicolumn{7}{c}{ Factors } \\
\cline { 2 - 8 } No. & $\begin{array}{c}\text { Printing } \\
\text { Speed }(\mathrm{mm} / \mathrm{s})\end{array}$ & $\begin{array}{c}\text { Nozzle } \\
\text { Temperature }\left({ }^{\circ} \mathrm{C}\right)\end{array}$ & $\begin{array}{c}\text { Bed Temperature } \\
\left({ }^{\circ} \mathrm{C}\right)\end{array}$ & Fill Percentage & $\begin{array}{c}\text { Nozzle } \\
\text { Diameter } \\
(\mathrm{mm})\end{array}$ & $\begin{array}{c}\text { Lamina } \\
\text { Thickness }(\mathrm{mm})\end{array}$ & $\begin{array}{c}\text { Extrusion } \\
\text { Multiplier }\end{array}$ \\
\hline 1 & 30 & 180 & 25 & 75 & 0.2 & 0.05 & 0.8 \\
2 & 30 & 180 & 25 & 100 & 0.5 & 0.2 & 1.2 \\
3 & 30 & 230 & 50 & 75 & 0.2 & 0.2 & 1.2
\end{tabular}




\begin{tabular}{cccccccc}
4 & 30 & 230 & 50 & 100 & 0.5 & 0.05 & 0.8 \\
5 & 120 & 180 & 50 & 75 & 0.5 & 0.05 & 1.2 \\
6 & 120 & 180 & 50 & 100 & 0.2 & 0.2 & 0.8 \\
7 & 120 & 230 & 25 & 75 & 0.5 & 0.2 & 0.8 \\
8 & 120 & 230 & 25 & 100 & 0.2 & 0.05 & 1.2 \\
\hline
\end{tabular}

$\mathrm{S} / \mathrm{N}$ ratio analysis indicates that the optimal condition is where the signal-to-noise ratio is maximum. The parameter of this analysis, $\eta$, is shown as follows and the more it changes for different levels of the input factor, the greater the effect of that factor on the output [37]:

$$
\eta=-10 \log _{10}\left[\frac{1}{s} \sum_{k=1}^{s}\left(\frac{1}{y_{k}^{2}}\right)\right]
$$

Where $y_{k}$ is the output value for $k$ th experiment and $\mathrm{s}$ is the number of iterations. Table 3 shows the values of $\eta$ and the output (strain), which was measured three times using DIC method. All samples were printed with a printing pattern of $[0]_{10}$ at room temperature. Figure 6 shows $\eta$ versus different printing parameters, based on which the parameters of layer thickness, nozzle temperature and print speed have a significant effect on the output and were chosen to further study in this paper.

Table 3. measured strains of each sample and its $S / N$ analysis parameter, $\eta$

\begin{tabular}{ccccc}
\hline Exp No. & \multicolumn{5}{c}{ Output (strain) } \\
& 1 & 2 & 3 & $\eta$ \\
\hline 1 & 0.346 & 0.351 & 0.342 & -9.21 \\
2 & 0.142 & 0.141 & 0.145 & -16.92 \\
3 & 0.029 & 0.031 & 0.029 & -30.57 \\
4 & 0.220 & 0.208 & 0.211 & -13.44 \\
5 & 0.452 & 0.418 & 0.422 & -7.33 \\
6 & 0.217 & 0.221 & 0.209 & -13.33 \\
7 & 0.092 & 0.095 & 0.091 & -20.67 \\
8 & 0.322 & 0.295 & 0.302 & -10.29 \\
\hline
\end{tabular}




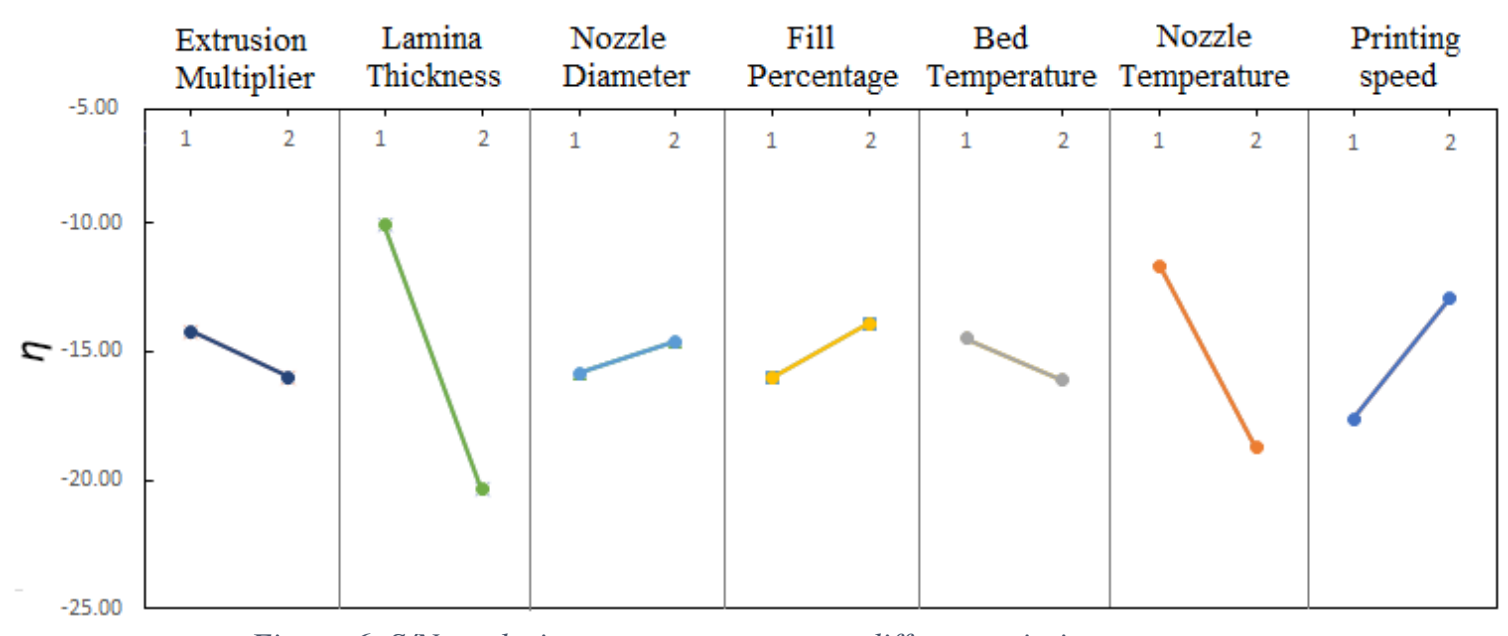

Figure 6. S/N analysis parameter, $\eta$, versus different printing parameters

After determining the effective parameters (i.e. Printing speed, Lamina thickness and Nozzle temperature), a full factorial design of experiments consisting three 4-level factors were performed to establish a relation between strain and printing parameters (see section 3.3). Factors and their levels are shown in Table 4. Note that each factor has a feasible range of values from which four levels were chosen in a way that the first and the last levels show the lower and upper limits of the range, respectively. Other printing parameters were set according to the values of printing parameters given in Figure 5 and the printing pattern of all samples were $[0]_{\mathrm{n}}$. All specimens $(40 \times 10 \times 1 \mathrm{~mm})$ were printed in triplicates.

Table 4. Factors and their levels used for full factorial DOE

\begin{tabular}{|c|c|c|c|c|}
\hline & & \multicolumn{3}{|c|}{ Factors $^{+}$} \\
\hline & & $\begin{array}{c}\text { Normalized Printing } \\
\text { speed }\left(v^{*}\right)\end{array}$ & $\begin{array}{c}\text { Normalized lamina } \\
\text { thickness }(h *)\end{array}$ & $\begin{array}{c}\text { Normalized nozzle } \\
\text { temperature }\left(T^{*}\right)\end{array}$ \\
\hline & 1 & 0.2 & 0.1 & 1.15 \\
\hline & 2 & 0.4 & 0.2 & 1.25 \\
\hline & 3 & 0.6 & 0.3 & 1.45 \\
\hline & 4 & 0.8 & 0.4 & 1.65 \\
\hline
\end{tabular}

\section{Model description}

Here we propose a simple but straightforward model to predict shape-shifting of a hinge; a model that enables user to change the magnitude of FDM process parameters as desired and get the resulting final shape-shifting behavior. Thus by controlling the shape-shifting of the hinges, this model is a mean to design 4D printed structures such as active origami.

3.1 Free recovery of a single layer 
To obtain the free recovery strain of a homogeneous thin beam of an SMP, constitutive equations were extracted using the Zener fraction model. Figure 7 shows a schematic of the Zener fraction model, which includes an equilibrium and a nonequilibrium branch. In this model, the dash-pot is replaced by an element termed a 'springpot' by Koeller [38]. This simple two branch fractional model has proven to be as accurate as a 12 branch generalized maxwell model or a five branch modified generalized maxwell model [39,40].

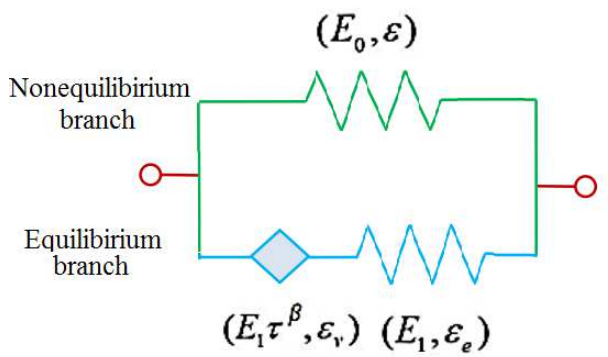

Figure 7. Schematic illustration of Zener fractional model

According to the mechanical model shown in Figure 7,

$$
\sigma=E_{0} \varepsilon+E_{1} \varepsilon_{e}=E_{0} \varepsilon+E_{1} \tau^{\beta} D^{\beta} \varepsilon_{v}, \quad \varepsilon=\varepsilon_{e}+\varepsilon_{v}
$$

The constitutive equation for the fractional Zener model can be written as:

$$
\left(1+\tau^{\beta} D^{\beta}\right) \sigma_{r}(t)=E_{0}\left(1+t_{1}^{\beta} D^{\beta}\right) \varepsilon_{r}(t)
$$

where $\sigma_{r}(t), \varepsilon_{r}(t), t, E$ and $\tau$ are the recovery stress, recovery strain, time, modulus of elasticity and relaxation time, respectively. $\beta$ is known as memory parameter or the fractional derivative parameter, and $D^{\beta}$ indicates fractional differentiation. Finally, $t_{1}=\tau \sqrt[\beta]{1+E_{1} / E_{0}}$.

By applying nonisothermal free recovery condition, and following the time temperature superposition principle (TTSP), the final form of the recovery strain for temperatures below reference temperature, $T_{0}\left(\right.$ or $\left.T_{L}+Q t<T_{0}\right)$, would be:

$$
\varepsilon_{r}(t)=e_{0} . \mathrm{E}_{\beta}\left[-\frac{E_{0}}{\left(E_{0}+E_{1}\right)} \int_{0}^{t} \frac{\beta \zeta^{\beta-1}}{\tau_{0}^{\beta}} \exp \left[\frac{\beta E_{a}}{2.303 R}\left(\frac{1}{T_{L}+Q \zeta}-\frac{1}{T_{0}}\right)\right] d \zeta\right]=e_{0} \cdot A(t)
$$

And as the temperature is raised above $T_{0}\left(\right.$ or $\left.T_{L}+Q t>T_{0}\right)$, we have:

$$
\varepsilon_{r}(t)=e_{0} \cdot\left(A\left(\frac{T_{0}-T_{L}}{Q}\right)+\mathrm{E}_{\beta}\left[-\frac{E_{0}}{\left(E_{0}+E_{1}\right)} \int_{\frac{T_{0}-T_{L}}{Q}}^{t} \frac{\beta \zeta^{\beta-1}}{\tau_{0}^{\beta}} \cdot 10^{\frac{\beta C_{1}\left(T_{L}+Q \zeta-T_{0}\right)}{C_{2}+\left(T_{L}+Q \zeta-T_{0}\right)}} d \zeta\right]\right)=e_{0} \cdot B(t)
$$

Where $C_{1}, C_{2}$ are material constants, $E_{a}$ is the configuration energy, $R$ is the gas constant, $Q$ is heating rate and $e_{0}$ is the strain stored in printed structure during printing process. $\varepsilon_{r}(t)$ defines the shape recovery strain, i.e. 
$\left.\varepsilon_{r}(t)\right|_{t=0}=e_{0}$, Therefore, the strain of a homogeneous thin beam of an SMP over time is obtained according to the following equation:

$$
\varepsilon(t)=e_{0}-\varepsilon_{r}(t)=e_{0}(1-B(t))=e_{0} R(t)
$$

$R(t)$ is the strain recovery ratio initially proposed by Leidlein [41].

There are nine parameters in Eqs. (4) \& (5) to be identified for prediction of free recovery of a single layer, which they are listed in Table 5. First five parameters namely $E_{0}, E_{1}, \beta, \tau_{0}, T_{0}$ where determined using [42]. To determine $C_{1}$ and $C_{2}$, DMA tests were performed in different frequencies of $0.5,1,10,20$, and $50 \mathrm{~Hz}$ and based on the TTSP, a master curve at reference temperature were constructed with shifting factors. finally to determine $E_{\mathrm{a}}$, tan delta curves for different frequencies were used (Figure 8.a). The $T_{\mathrm{g}}$ at different frequencies is determined from the peak of the tan delta curves and $E_{\mathrm{a}}$ is calculated from the slope of the plot of $\ln (f)$ versus $1 / T_{\mathrm{g}}$ (Figure $8 . \mathrm{b}$ ) using the following equation [43]:

$$
\mathrm{E}_{a}=-R \frac{d(\ln (f))}{d\left(1 / T_{g}\right)}
$$

Where $f$ is frequency $(\mathrm{Hz})$. After identifying all parameters, now we can use Eqs. (4) \& (5) to predict free recovery strain of each layer through time and temperature. Figure 9 compares the model predictions and experimental data for strain recovery ratio. As it can be seen, the results of the model has a good agreement with experiments.

Table 5. Parameters for free recovery strain model prediction

\begin{tabular}{ccc}
\hline Parameter & Value & Description \\
\hline$E_{0}(\mathrm{Mpa})$ & 200 & Elastic modulus in the equilibrium branch \\
$E_{1}(\mathrm{Mpa})$ & 3000 & Elastic modulus in the nonequilibrium branch \\
$\beta(-)$ & 0.35 & Fractional derivative parameter \\
$\tau_{0}(\mathrm{~s})$ & 71429 & Relaxation time at reference temperature \\
$T_{0}\left({ }^{\circ} \mathrm{C}\right)$ & 35 & Reference temperature \\
$C_{1}(-)$ & 16.5 & Parameter of the WLF equation \\
$C_{2}\left({ }^{\circ} \mathrm{C}\right)$ & 55 & Parameter of the WLF equation \\
$E_{a}(\mathrm{~kJ} / \mathrm{mol})$ & 502 & Configuration energy \\
$R(\mathrm{~J} / \mathrm{K} / \mathrm{mol})$ & 8.314 & Gas constant \\
\hline
\end{tabular}



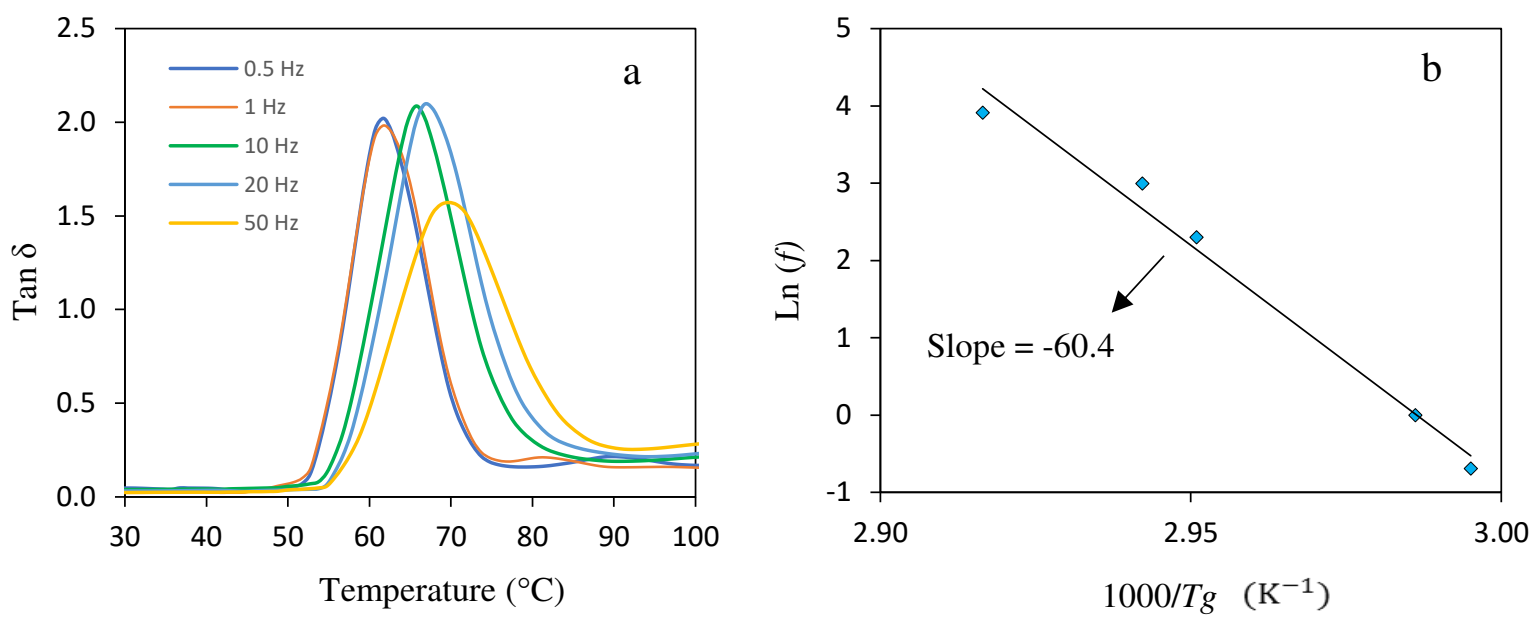

Figure 8. a) Tan $\delta$ curves for a range of frequencies, b) Frequency in a log scale vs the inverse of glass transition temperature

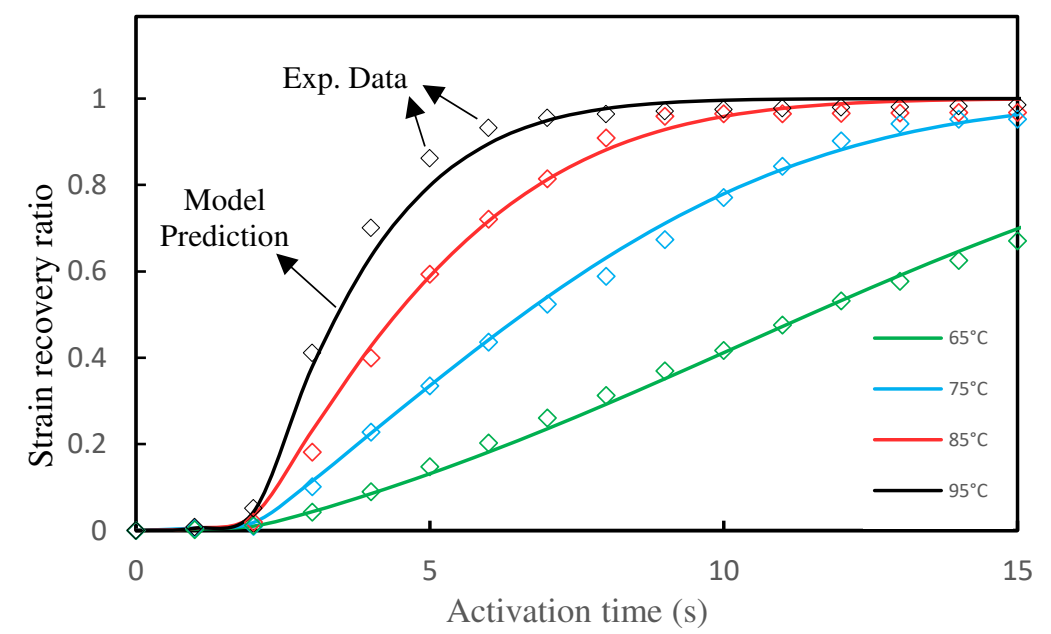

Figure 9. Comparison between model predictions and experimental data for strain recovery ratio

\subsection{Relation between stored strain and printing pattern}

A single lamina printed with straight parallel rasters exhibits obvious mechanical anisotropy along the extrusion direction and the perpendicular direction as printing process cause polymer chains to stretch and align along the direction of extrusion, resembling the fiber and matrix in Uni-Directional composite plies. Based on this assumption, we used Transformation matrix to relate the strain of angle laminae to strain of the laminae with printing pattern of $[0]_{z}$. The coordinate system used for showing an angle lamina is given in Figure 10. The axes in the 1-2 coordinate system are called the local axes and the axes in the $x-y$ coordinate system are called the global axes. On this basis, strain of an angle lamina is obtained as follows [44]: 


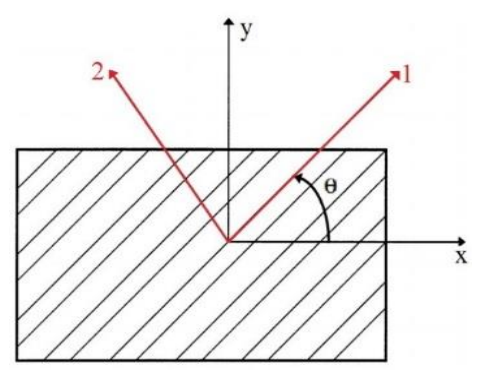

Figure 10. Local and global axes of an angle lamina

$$
e_{0}=\left[\begin{array}{c}
\varepsilon_{x} \\
\varepsilon_{y} \\
\gamma_{x y}
\end{array}\right]
$$

$$
\left[\begin{array}{c}
\varepsilon_{x} \\
\varepsilon_{y} \\
\gamma_{x y}
\end{array}\right]=[R][T]^{-1}[R]^{-1}\left[\begin{array}{c}
\varepsilon_{1} \\
\varepsilon_{2} \\
\gamma_{12}
\end{array}\right]
$$

Where $[T]$ is the Transformation matrix and $[R]$ is the Reuter matrix and are defined as:

$$
\begin{array}{ll}
{[T]=\left[\begin{array}{ccc}
c^{2} & s^{2} & 2 s c \\
s^{2} & c^{2} & -2 s c \\
-s c & s c & c^{2}-s^{2}
\end{array}\right]} & , \quad[R]=\left[\begin{array}{lll}
1 & 0 & 0 \\
0 & 1 & 0 \\
0 & 0 & 2
\end{array}\right] \\
c=\cos (\Theta) \quad, \quad s=\sin (\Theta) &
\end{array}
$$

Assuming linear distribution of strain through thickness, and using superposition principle, the strain of a layer consisting of $z$ laminae is:

$$
\left[\begin{array}{c}
\varepsilon_{x} \\
\varepsilon_{y} \\
\gamma_{x y}
\end{array}\right]=\frac{1}{z} \sum_{i=1}^{z}[\bar{T}]_{i}\left[\begin{array}{c}
\varepsilon_{1} \\
\varepsilon_{2} \\
\gamma_{12}
\end{array}\right]
$$

Where $[\bar{T}]_{i}$ is the effective transformation matrix for $i$ th lamina with an angle of $\Theta_{i}$ and is defined as:

$$
[\bar{T}]_{i}=\left[\begin{array}{ccc}
c^{2}{ }_{i} & s^{2}{ }_{i} & -s_{i} c_{i} \\
s_{i}{ }^{2} & c_{i}{ }^{2} & s_{i} c_{i} \\
2 s_{i} c_{i} & -2 s_{i} c_{i} & c^{2}{ }_{i}-s^{2}{ }_{i}
\end{array}\right] \quad c_{i}=\cos \left(\Theta_{i}\right) \quad, \quad s_{i}=\sin \left(\Theta_{i}\right)
$$

\subsection{Relation between stored strain and printing parameters}

Since the strain will be stored in printed layer during the printing process, It is obvious that printing parameters namely printing speed, layer thickness and nozzle temperature will directly impact the strain. Considering that the effect of several variables were examined simultaneously, Multiple Linear Regression (MLR) technique was used to find the strain relationship (appendix I). Before using the technique, we made the abovementioned printing parameters dimensionless respectively as follows: $v^{*}=\frac{v}{v_{\max }}, h^{*}=\frac{h_{l}}{D_{n}}$ and $T^{*}=\frac{T_{n}-T_{g}}{T_{m}-T_{g}}$, Where $v_{\max }$ is nominal 
printing speed $\left(150 \mathrm{~mm} / \mathrm{s}\right.$ in this study), $D_{n}$ is nozzle diameter $\left(0.5 \mathrm{~mm}\right.$ in this study), $h_{l}$ is lamina thickness, and $T_{m}$ is melting temperature. Now according to the MLR technique we have:

$$
\left[\begin{array}{c}
\varepsilon_{1} \\
\varepsilon_{2} \\
\gamma_{12}
\end{array}\right]=[X]\left[\begin{array}{c}
v^{*} \\
h^{*} \\
T^{*}
\end{array}\right]+[Y]
$$

Where $\varepsilon_{1}, \varepsilon_{2}, \gamma_{12}$ are longitudinal, transverse and shear strains, respectively. $[X]$ and $[Y]$ are the coefficient and constant value matrices respectively. After minimizing the residual sum of squares (RSS), full factorial design of experiment was performed (Appendix I, table I-1) to extract these coefficients, which are given in equation (I-5).

\subsection{Curvature modelling of hinges}

According to Timoshenko [36], a beam made of two layers of metal with different coefficients of thermal expansion (CTE), will bend if heated due to the strain difference created in the layers. Figure 11 shows a bilayer beam which undergoes bending over activation. Considering the fact that here each of the two layers forming the hinge is of the same material and differs only in printing conditions, the difference in CTE of the layers is neglectable; moreover, Timoshenko's theory is for solids. For these reasons, it cannot predict the curvature of the self-bending hinges that have viscoelastic properties. However, the prediction of the curvature is expected via modification of the relationship for SMPs.
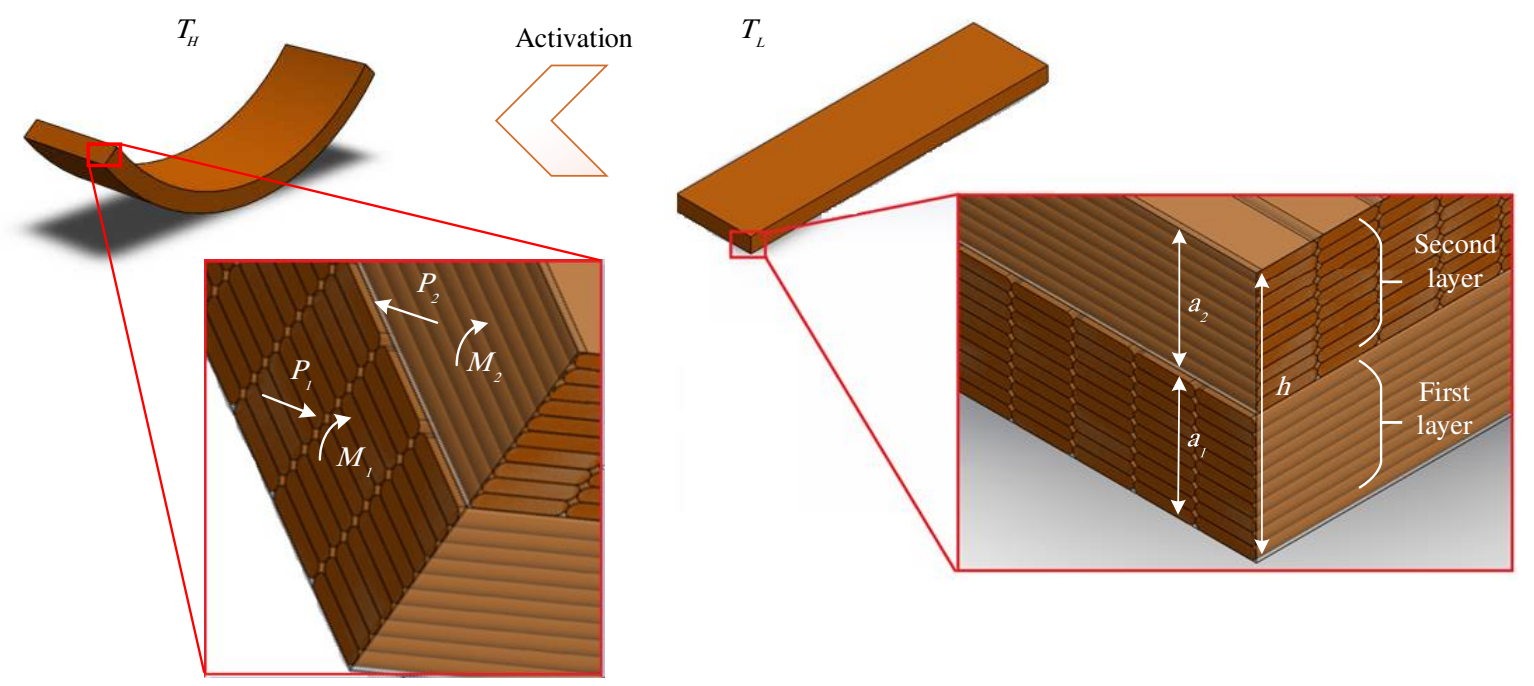

Figure 11. Bending of a bilayer hinge over activation (uniformly heated)

At the interface of the two layers, strain can be written as below:

$$
\begin{aligned}
\left(\varepsilon_{\text {thermal }}+\varepsilon_{\text {stress }}\right. & \left.+\varepsilon_{\text {curvature }}+\varepsilon_{\text {shape memory }}\right)_{l 1} \\
& =\left(\varepsilon_{\text {thermal }}+\varepsilon_{\text {stress }}+\varepsilon_{\text {curvature }}+\varepsilon_{\text {shape memory }}\right)_{l 2}
\end{aligned}
$$


So:

$$
\alpha_{1}\left(t-t_{0}\right)-\frac{P_{1}}{E_{1} a_{1}}-\frac{a_{1}}{2 \rho}+\varepsilon(t)_{l 1}=\alpha_{2}\left(t-t_{0}\right)+\frac{P_{2}}{E_{2} a_{2}}+\frac{a_{2}}{2 \rho}+\varepsilon(t)_{l 2}
$$

Assuming $\frac{a_{1}}{a_{2}}=m, \frac{E_{1}}{E_{2}}=n$ and $\alpha_{1} \approx \alpha_{2}$, curvature would be:

$$
\kappa(t)=\frac{6\left(\varepsilon(t)_{l 1}-\varepsilon(t)_{l 2}\right)(1+m)^{2}}{h\left(3(1+m)^{2}+(1+m n)\left(m^{2}+\frac{1}{m n}\right)\right)}
$$

The Curvature could be in $x$ or $y$ direction:

$$
\kappa_{i}(t)=\left[\frac{6(1+m)^{2}}{h\left(3(1+m)^{2}+(1+m n)\left(m^{2}+\frac{1}{m n}\right)\right)}\right]\left(\varepsilon_{i}(t)_{l 2}-\varepsilon_{i}(t)_{l 1}\right) \quad, \quad i=x, y
$$

The modulus of elasticity must be obtained for each layer to calculate the value of $n$. Assuming the printed rasters as fibers in composites, elastic modulus for different printing patterns has been modeled by [30] using CLT.

It can be shown that when the thickness of the two layers are the same, i.e. $m=1, \mathrm{n}$ does not produce any substantial effect on curvature[36], thus, Eq (17) can be rewritten as:

$$
\kappa_{i}(t)=\frac{3}{2 h}\left(\varepsilon_{i}(t)_{l 2}-\varepsilon_{i}(t)_{l 1}\right) \quad, \quad i=x, y
$$

\subsection{Torsion modelling of hinges}

Timoshenko's constitutive equations for beam torsion are used to derive the torsion of a two-layer structure.

Figure 12 shows a bilayer beam which undergoes twisting over activation. All the forces acting over the section of the first layer can be represented by a shear force $V_{1}$ and a torsional moment $V_{1}$. For the second layer, All forces acting on the cross-section can be represented by a shear force $V_{2}$ and a torsional moment $T_{2}$. Since no external force is applied to the beam, all forces acting on any cross section of the beam must be in equilibrium. 

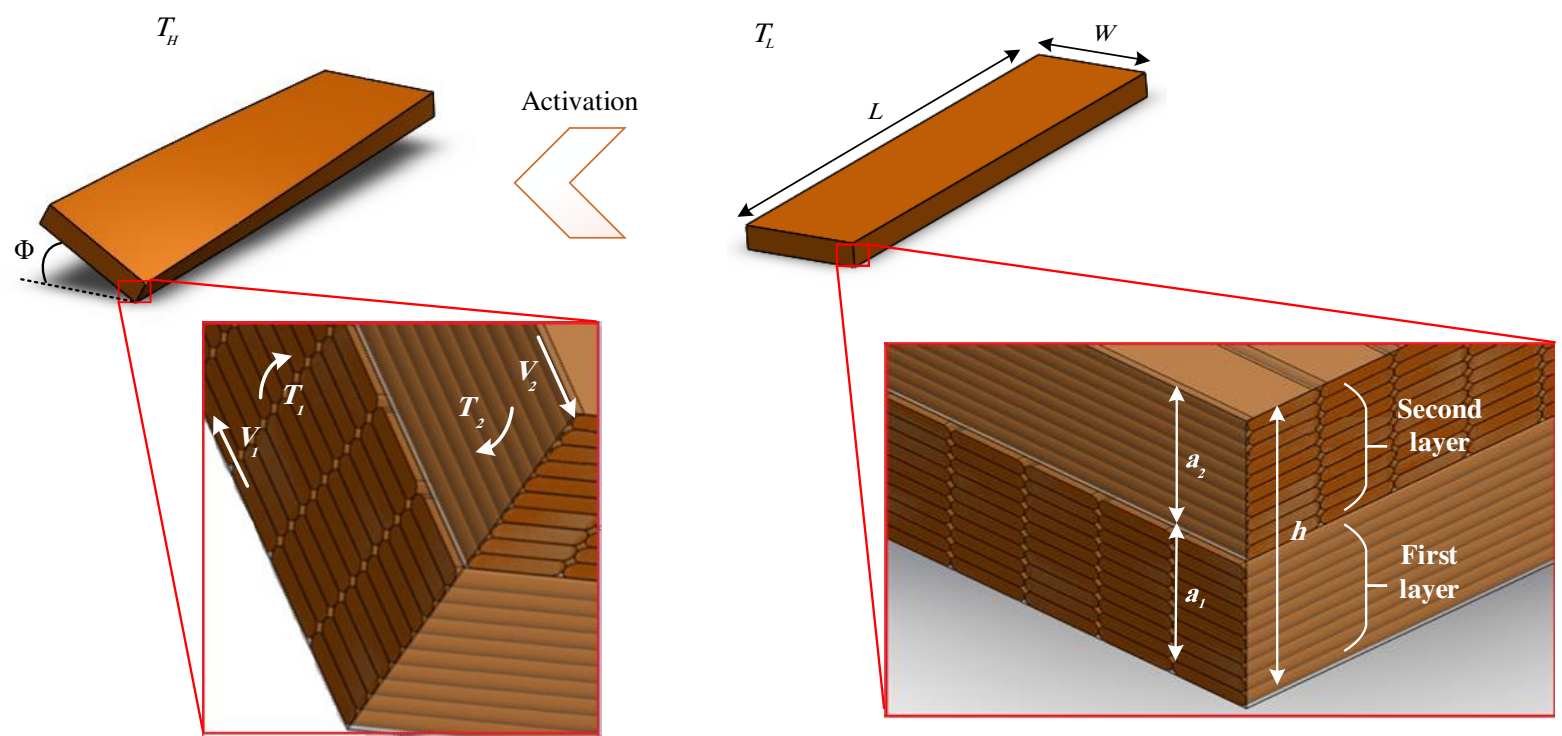

Figure 12. twisting of a bilayer hinge over activation (uniformly heated)

At the interface of the two layers, shear strain can be written as below:

$$
\left(\gamma_{\text {twist }}+\gamma_{\text {stress }}+\gamma_{\text {shape memory }}\right)_{l 1}=\left(\gamma_{\text {twist }}+\gamma_{\text {stress }}+\gamma_{\text {shape memory }}\right)_{l 2}
$$

Thus:

$$
\frac{\kappa_{x y} W}{2}+\frac{V_{1}}{G_{1} a_{1} W}+\left(\gamma_{12}(t)\right)_{l 1}=-\frac{\kappa_{x y} W}{2}-\frac{V_{2}}{G_{2} a_{2} W}+\left(\gamma_{12}(t)\right)_{l 2}
$$

Assuming $\frac{a_{1}}{a_{2}}=m, \frac{G_{1}}{G_{2}}=\dot{n}, \frac{W}{h}=\dot{w}$, the final torsion where strain recovery ratio tends to one, would be:

$$
\kappa_{x y}(t)=\left[\frac{3(1+m)^{2}}{h\left((3 \dot{w}+1)(1+m)^{2}+\left(1+m n^{\prime}\right)\left(m^{2}+\frac{1}{m n}\right)\right)}\right]\left(\gamma_{x y}(t)_{l 2}-\gamma_{x y}(t)_{l 1}\right)
$$

Like curvature, It can be shown that when the thickness of the two layers are the same, $n$ does not produce any substantial effect on torsion, thus, Eq (21) can be rewritten as:

$$
\kappa_{x y}=\frac{3}{h(3 \dot{w}+2)}\left(\gamma_{x y}(t)_{l 2}-\gamma_{x y}(t)_{l 1}\right)
$$

\section{Results and discussion}

\section{1 stored strain and printing pattern}

To verify Equation (12), samples with different printing patterns (angles) were printed and their longitudinal, transverse and shear strains were measured carefully after activation. Figure 13 shows good agreement between the experimental data and predictions of the model. The printing conditions are given in Figure 5. 

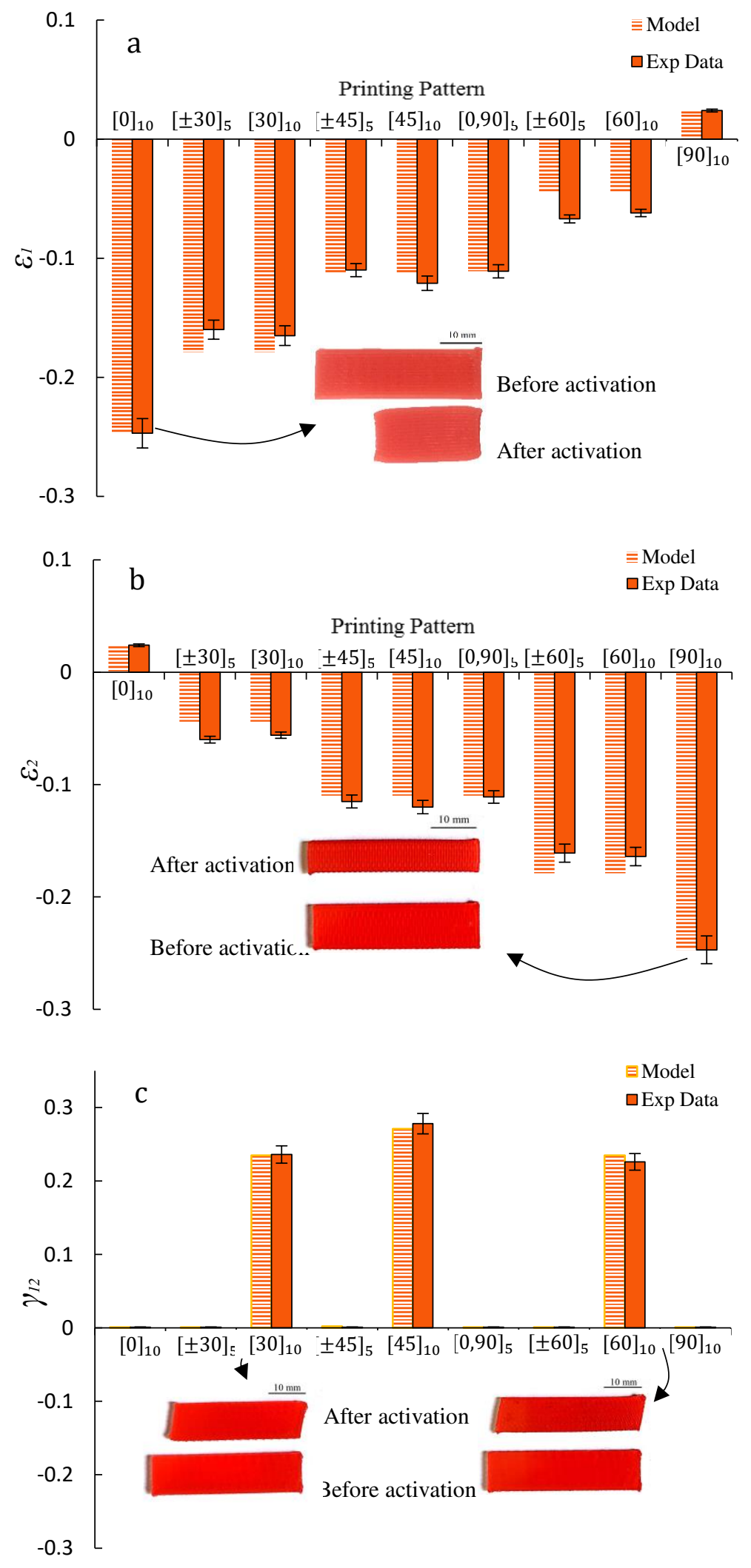

Figure 13.(a) longitudinal,(b) transverse and (c) shear Strains of printed specimens with different printing patterns (angles) in comparison with model predictions 


\section{2 modification of curvature and torsion of bilayer beams}

The model predictions for curvature and torsion are expected to differ from the experimental results by an extent, necessitating the modification of the model. There are two major reasons behind this difference: first, the calculated free recovery strain of a single layer will be different from that of the same layer while in a bilayer structure as it is constrained by the next layer, and second, the other dimensions of each layer such as thickness and width, will also undergo a different amount of strain through the activation process which consequently alters the value of $w, m$ and the total thickness, $h$, in addition. For the sake of model simplicity, we won't apply these conditions to the model and instead will add a correction factor using experimental data. Process parameters are intentionally set in a way that the resulting strains increase incrementally.

Figure 14.a compares the experimental data of the final curvature, $\kappa_{i}$ which is at $R(t) \approx 1$, with its predicted value by the proposed model for different values of $\Delta \varepsilon_{i}$ (longitudinal and transverse strain differences between two layers). The slope of the graph as shown in Figure 14.b is the correction factor $\alpha=0.67$ for curvature. As for torsion, Figure 15.a compares the experimental data of the final torsion, $\kappa_{x y}$, with its predicted value by the proposed model for different values of $\Delta \gamma_{x y}$ (shear strain differences between two layers). Here slope of the graph as shown in Figure 15.b would be the correction factor $\hat{\beta}=1.4$ for torsion.
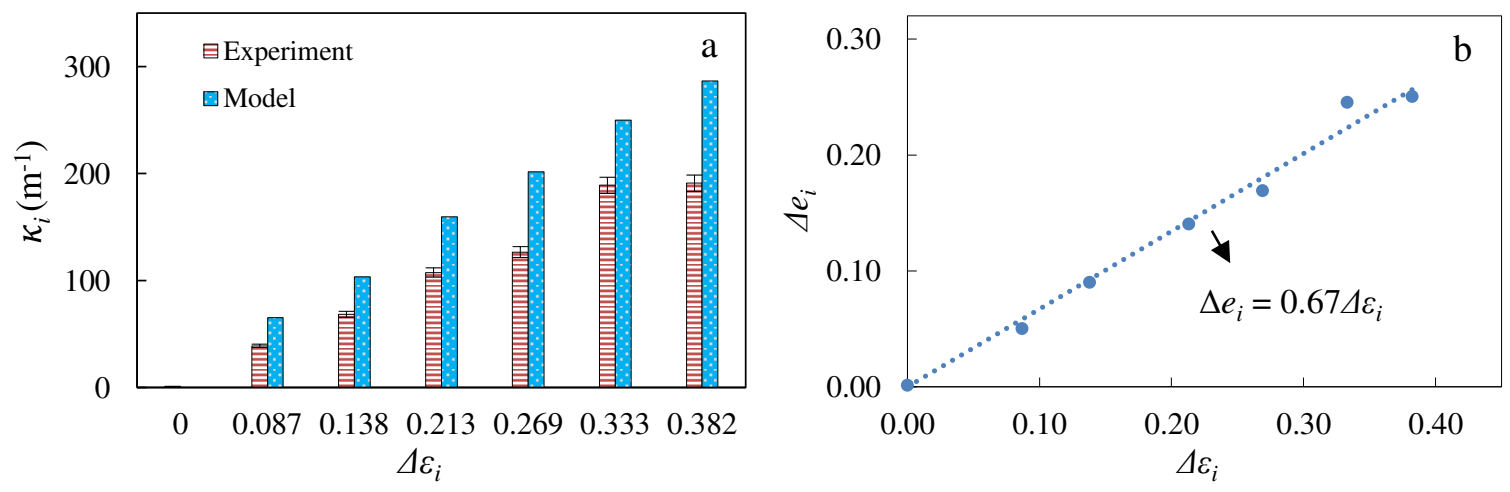

Figure 14. (a) Comparison of experimental data on the final curvature and its predicted value by proposed model, (b) Model predictions of two layers longitudinal/transverse strain difference versus that of experimental results. 

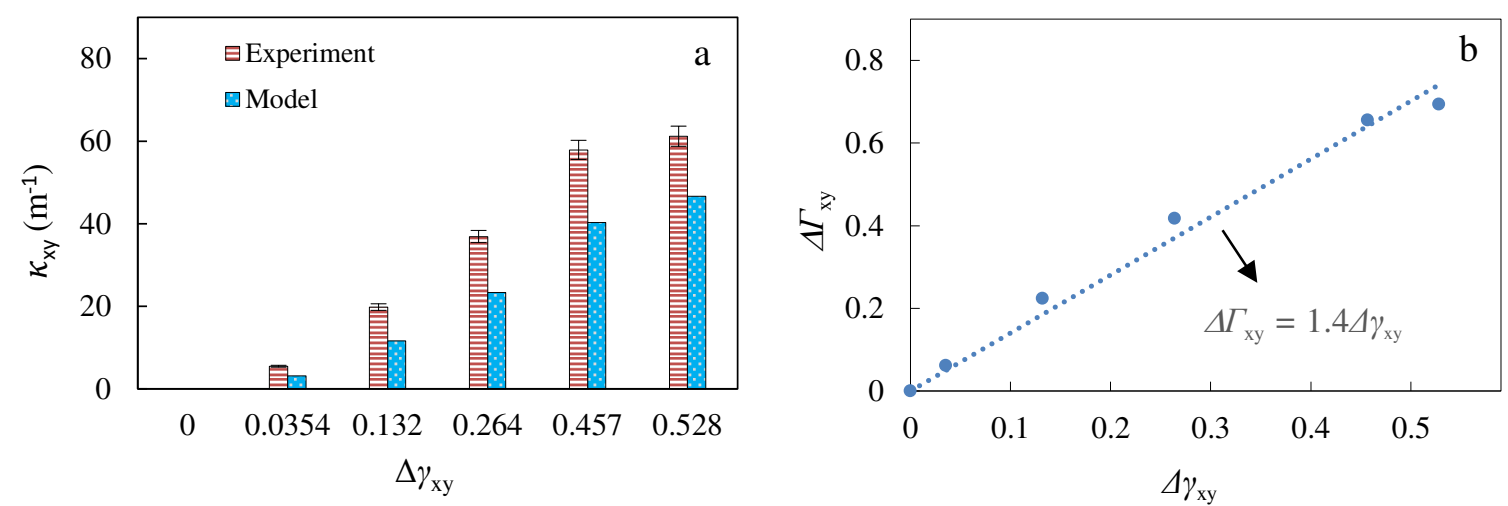

Figure 15. (a)Comparison of experimental data on the final twist and its predicted value by proposed model, (b) Model predictions of two layers shear strain difference versus that of experimental results.

These correction factors are extracted for PLA. To evaluate the validity of these correction factors, samples of ABS polymer were printed with the same conditions as PLA samples and the results as given in Figure 16 ( $\alpha=0.71$ and $\dot{\beta}=1.34$ ) showed good agreement with PLA correction factors.
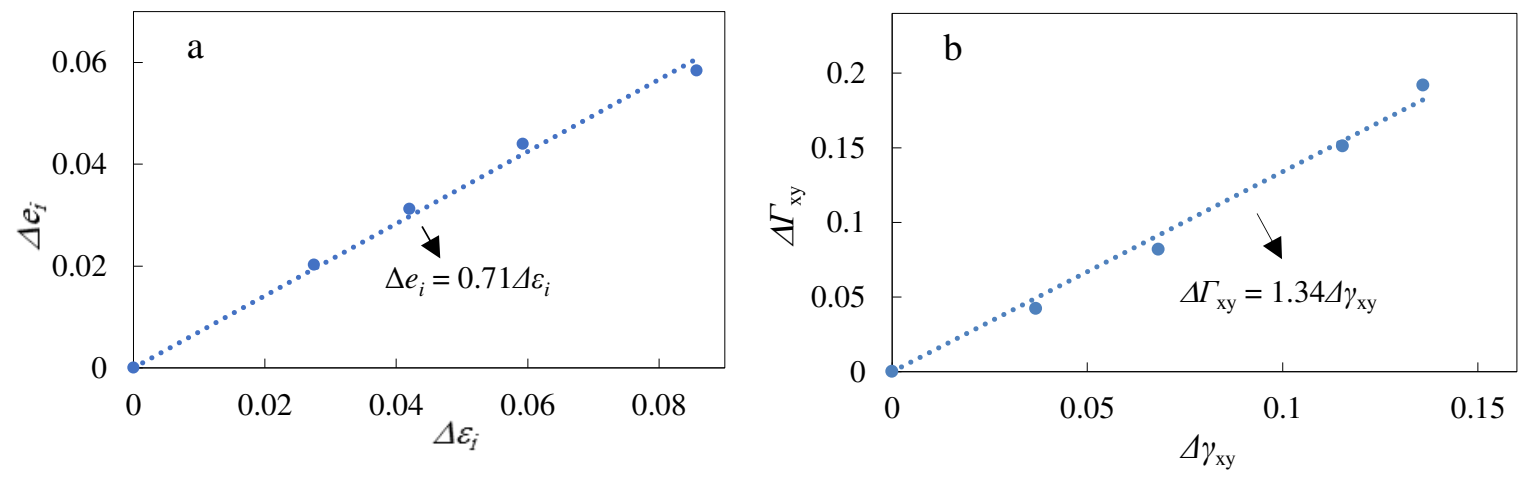

Figure 16. Model predictions of two layers strain difference versus that of experimental results for ABS.

\subsection{D printed bilayer shape shifting model}

By combining Eqs (6), (11), (13), (17) and (21), the curvature and torsion relations of bilayer 4D printed structures can be written in a matrix form:

$$
\left[\begin{array}{c}
\kappa_{x} \\
\kappa_{y} \\
\kappa_{x y}
\end{array}\right]=[C]\left([X]\left[\begin{array}{c}
v^{*} \\
h^{*} \\
T^{*}
\end{array}\right]+[Y]\right) \quad \text { or } \quad\left[\begin{array}{c}
\kappa_{x} \\
\kappa_{y} \\
\kappa_{x y}
\end{array}\right]=[C]\left[\begin{array}{c}
\varepsilon_{1} \\
\varepsilon_{2} \\
\gamma_{12}
\end{array}\right]
$$

Where matrix $[C]$ is defined as:

$C_{i j}=\frac{6 \dot{\alpha}(1+m)^{2}}{h z\left(3(1+m)^{2}+(1+m n)\left(m^{2}+\frac{1}{m n}\right)\right)}\left[\sum_{k=1}^{\frac{m z}{m+1}} \bar{T}_{i j}-\sum_{k=\frac{m z}{m+1}+1}^{\frac{z}{m+1}} \bar{T}_{i j}\right], \quad i=1,2 ; \quad j=1,2,3$ 
$C_{i j}=\frac{3 \hat{\beta}(1+m)^{2}}{h z\left((3 \dot{w}+1)(1+m)^{2}+(1+m n)\left(m^{2}+\frac{1}{m n}\right)\right)}\left[\sum_{k=1}^{\frac{m z}{m+1}} \bar{T}_{i j}-\sum_{k=\frac{m z}{m+1}+1}^{\frac{z}{m+1}} \bar{T}_{i j}\right], \quad i=3 ; \quad j=1,2,3$

If the thickness of the two layers is equal which in most cases they are, Eq (25) can be rewritten as:

$C_{i j}=\frac{3 \dot{\alpha}}{2 h z}\left[\sum_{k=1}^{z / 2} \bar{T}_{i j}-\sum_{k=z / 2}^{z} \bar{T}_{i j}\right], \quad i=1,2 ; \quad j=1,2,3$

$C_{i j}=\frac{3 \dot{\beta}}{h z(3 \dot{w}+2)}\left[\sum_{k=1}^{z / 2} \bar{T}_{i j}-\sum_{k=z^{z} / 2^{+1}}^{z} \bar{T}_{i j}\right], \quad i=3 ; \quad j=1,2,3$

\subsection{Model verification}

According to the application of these bilayer structures, $\kappa_{y}$ is not considered and to verify the accuracy of the model, only the predicted values of $\kappa_{x}$ and $\kappa_{x y}$ were compared with xperimental data. As shown in Figure 17, three samples were designed and printed in such a way that the sample (a) goes under pure bending, sample (b) pure torsion and sample (c) shows Both bending and twisting shape shifting behaviors. The printing conditions of the three samples are shown in Table 6 . Activation time and temperature for all samples $(40 \times 10 \times 2 \mathrm{~mm})$ were set to $90^{\circ} \mathrm{C}$ and 30 seconds, respectively.

As it can be seen in Figure 17, model results show good agreement with experimental data, thus model is capable of predicting the final shape shifting behavior and it's magnitude.

a

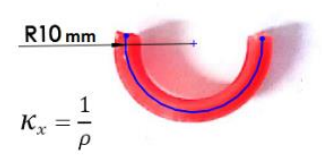

\begin{tabular}{ccc}
\hline & Exp Data & Model \\
\hline$\kappa_{x}\left(\mathrm{~m}^{-1}\right)$ & $99 \pm 5$ & 95 \\
$\kappa_{x y}\left(\mathrm{~m}^{-1}\right)$ & 0 & 0 \\
\hline
\end{tabular}

b
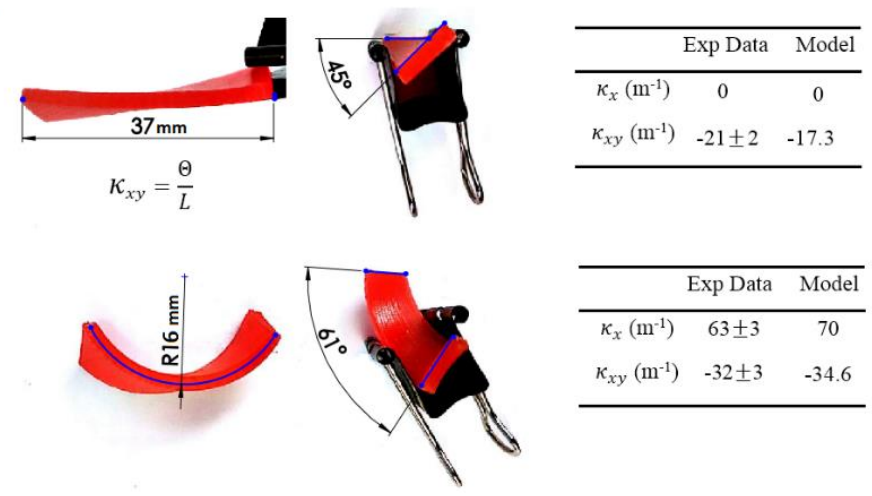

Figure 17. Comparison of model results with experimental data on final shape shifting behavior of hinges 
Table 6. Printing conditions of the verification samples

\begin{tabular}{lccccc}
\hline Sample No. & $\boldsymbol{v}^{*}$ & $\boldsymbol{h}^{*}$ & $\boldsymbol{T}^{*}$ & Print \\
& & & & pattern \\
\hline & 1st layer & 0.6 & 0.2 & 1.15 & {$[0]_{10}$} \\
\hline & 2nd layer & 0.6 & 0.2 & 1.15 & {$[ \pm 45]_{5}$} \\
\hline b & 1st layer & 0.6 & 0.2 & 1.45 & {$[ \pm 15]_{5}$} \\
& 2nd layer & 0.6 & 0.2 & 1.45 & {$[15]_{10}$} \\
\hline & 1st layer & 0.6 & 0.2 & 1.45 & {$[0]_{5}$} \\
& & & & & \\
\hline
\end{tabular}

\subsection{Discussion}

It was shown that a single layer 4D printed SMP beam would shrink to an extend after being activated in higher temperatures than its $T_{g}$. Printing a bilayer beam with each layer having a different strain, would cause out of plane shape shifting behaviors like bending and twisting. In order to have two layers with different strains, 3D printing process parameters should be changed for each layer. We have experimentally determined the parameters with significant effect on strains. Then a model were developed based on that parameters to predict the shape shifting behaviors and their magnitude. According to the proposed model, printing parameters affect the magnitude of the strains, whereas the printing pattern affect the direction of the stains, also difference in longitudinal and transverse strains will cause bending behavior in $\mathrm{x}$ and $\mathrm{y}$ directions respectively, and difference in shear strains will cause twisting behavior in bilayer structure. To achieve both behaviors simultaneously, both longitudinal/transverse strains and shear strains of the two layers should be different. In this regard, a shapeshifting flowchart as shown in Figure 18 were designed so that one can achieve desired shape-shifting behavior by following this flowchart. 


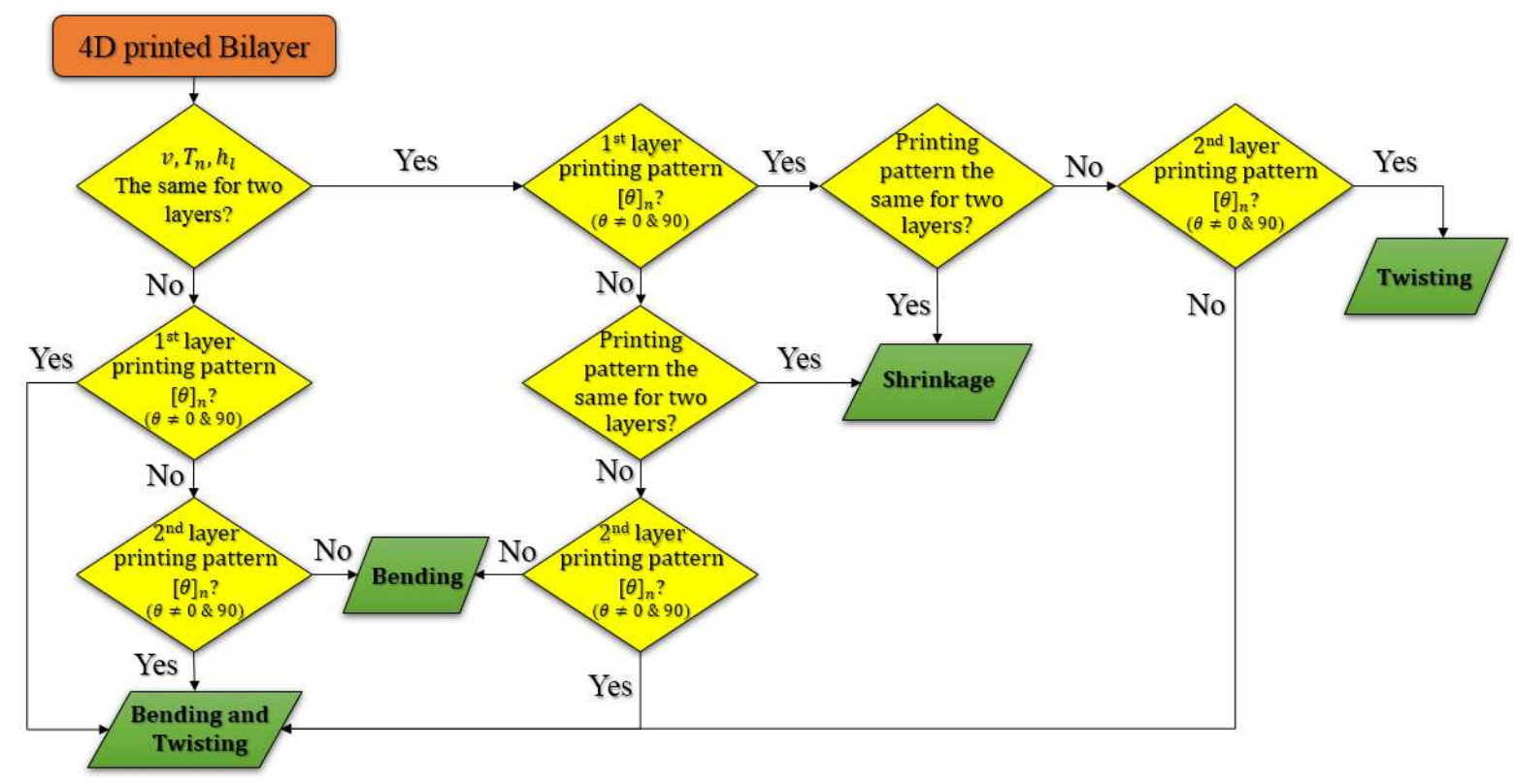

Figure 18. shape-shifting behavior flowchart for 4D printed bilayer strucures (hinges)

\section{Conclusions}

In this paper, we developed a semi-empirical model to control and predict the shape-shifting behavior of the bilayer structures aka hinges which printed using FDM 3D printing process. Our model allows the user to desirably change the process parameters including printing parameters and patterns and see the resulting shape shifting behavior and its magnitude skipping the trial and error experiments. The results of the model shows a good agreement with experiment data and is accurate enough to be used in practice. The model also shows the effective 3D printing parameters and their impact on shape shifting magnitude where increasing the print speed increases it and decreasing the nozzle temperature and layer heigh decrease it. The next step would be to predict the shapeshifting behavior of the hinges through the activation time. In addition, model can predict the shape-shifting behavior of the hinges through activation time and temperature. By predicting the behavior through time, one can design multiple and/or multi-step desired shapes, not to mention the further control over the shape-shifting magnitude.

\section{Declarations}

\section{Funding}

No funding was received to assist with the preparation of this manuscript. 


\section{Conflicts of interest/Competing interests}

The authors have no conflicts of interest to declare that are relevant to the content of this article.

\section{Availability of data and material}

All data used in this work will be presented upon reasonable request.

\section{Code availability}

Not applicable

\section{Ethics approval}

Not applicable

\section{Consent to participate}

Not applicable

\section{Consent for publication}

Not applicable

\section{REFERENCES}

[1] Tibbits S. The emergence of “4D printing.” TED Conf., 2013.

[2] Tibbits S. 4D printing: Multi-material shape change. Archit Des 2014;84:116-21. https://doi.org/10.1002/ad.1710.

[3] Tibbits S, McKnelly C, Olguin C, Dikovsky D, Hirsch S. 4d Printing and Universal Transformation. Proc. 34th Annu. Conf. Assoc. Comput. Aided Des. Archit., 2014, p. 539-48.

[4] Zhang Y, Zhang F, Yan Z, Ma Q, Li X, Huang Y, et al. Printing, folding and assembly methods for forming 3D mesostructures in advanced materials. Nat Rev Mater 2017;2:17019. https://doi.org/10.1038/natrevmats.2017.19.

[5] Teoh JEM, An J, Feng X, Zhao Y, Chua CK, Liu Y. Design and 4D printing of cross-folded origami structures: A preliminary investigation. Materials (Basel) 2018;11. https://doi.org/10.3390/ma11030376.

[6] Ding Z, Yuan C, Peng X, Wang T, Qi HJ, Dunn ML. Direct 4D printing via active composite materials. Sci Adv 2017;3:e1602890. https://doi.org/10.1126/sciadv.1602890.

[7] Wu J, Yuan C, Ding Z, Isakov M, Mao Y, Wang T, et al. Multi-shape active composites by 3D printing of digital shape memory polymers. Sci Rep 2016;6:24224. https://doi.org/10.1038/srep24224. 
[8] Mao Y, Yu K, Isakov MS, Wu J, Dunn ML, Jerry Qi H. Sequential Self-Folding Structures by 3D Printed Digital Shape Memory Polymers. Sci Rep 2015;5:13616. https://doi.org/10.1038/srep13616.

[9] Wei H, Zhang Q, Yao Y, Liu L, Liu Y, Leng J. Direct-Write Fabrication of 4D Active Shape-Changing Structures Based on a Shape Memory Polymer and Its Nanocomposite. ACS Appl Mater Interfaces 2017;9:876-83. https://doi.org/10.1021/acsami.6b12824.

[10] Choong YYC, Maleksaeedi S, Eng H, Wei J, Su PC. 4D printing of high performance shape memory polymer using stereolithography. Mater Des 2017;126:219-25. https://doi.org/10.1016/j.matdes.2017.04.049.

[11] Ge Q, Sakhaei AH, Lee H, Dunn CK, Fang NX, Dunn ML. Multimaterial 4D Printing with Tailorable Shape Memory Polymers. Sci Rep 2016;6:31110. https://doi.org/10.1038/srep31110.

[12] Kokkinis D, Schaffner M, Studart AR. Multimaterial magnetically assisted 3D printing of composite materials. Nat Commun 2015;6:8643. https://doi.org/10.1038/ncomms9643.

[13] Naficy S, Gately R, Gorkin R, Xin H, Spinks GM. 4D Printing of Reversible Shape Morphing Hydrogel Structures. Macromol Mater Eng 2017;302:1-9. https://doi.org/10.1002/mame.201600212.

[14] Wang W, Yuk C, Antonio P, Serrano A, Ahn S. Soft grasping mechanisms composed of shape memory polymer based self- bending units. Compos Part B 2019;164:198-204. https://doi.org/10.1016/j.compositesb.2018.10.081.

[15] Liu Y, Zhang W, Zhang F, Lan X, Leng J, Liu S, et al. Shape memory behavior and recovery force of 4D printed laminated Miura- origami structures subjected to compressive loading. Compos Part B 2018;153:233-42. https://doi.org/10.1016/j.compositesb.2018.07.053.

[16] Hu GF, Damanpack AR, Bodaghi M, Liao WH. Increasing dimension of structures by 4D printing shape memory polymers via fused deposition modeling. Smart Mater Struct 2017;26.

[17] Bodaghi M, Serjouei A, Zolfagharian A, Fotouhi M, Rahman H, Durand D. Reversible energy absorbing meta-sandwiches by FDM 4D printing. Int J Mech Sci 2020;173. https://doi.org/10.1016/j.ijmecsci.2020.105451.

[18] Teoh JEM, Zhao Y, An J, Chua CK, Liu Y. Multi-stage Responsive 4D Printed Smart Structure through Varying Geometric Thickness of Shape Memory Polymer. Smart Mater Struct 2017;26. https://doi.org/10.1088/1361-665X/aa8886.

[19] Jamshidi M, Salimi Nezhad I, Golzar M. Investigation of the Effect of 3D printing parameters on shapeshifting of flat sturctures to Three-Dimensional Shapes. J Sci Technol Compos 2021;07:1271-8.

[20] van Manen T, Janbaz S, Zadpoor AA. Programming 2D/3D shape-shifting with hobbyist 3D printers. Mater Horiz 2017. https://doi.org/10.1039/C7MH00269F.

[21] Yamamura S, Iwase E. Hybrid hinge structure with elastic hinge on self-folding of 4D printing using a fused deposition modeling 3D printer. Mater Des 2021;203:109605. 
https://doi.org/10.1016/j.matdes.2021.109605.

[22] Wang Y, Li X. An accurate finite element approach for programming 4D-printed self-morphing structures produced by fused deposition modeling. Mech Mater 2020;151:103628. https://doi.org/10.1016/j.mechmat.2020.103628.

[23] Carrell J, Gruss G, Gomez E. Four-dimensional printing using fused-deposition modeling: a review. Rapid Prototyp J 2020;26:855-69. https://doi.org/10.1108/RPJ-12-2018-0305.

[24] Tao Y, Gu J, An B, Cheng T. Demonstrating Thermorph : Democratizing 4D Printing of Self-Folding Materials and Interfaces 2018:1-4.

[25] Momeni F, M.Mehdi Hassani.N S, Liu X, Ni J. A review of 4D printing. Mater Des 2017;122:42-79. https://doi.org/10.1016/j.matdes.2017.02.068.

[26] Ge Q, Dunn CK, Qi HJ, Dunn ML. Active origami by 4D printing. Smart Mater Struct 2014;23:094007. https://doi.org/10.1088/0964-1726/23/9/094007.

[27] Melocchi A, Inverardi N, Uboldi M, Baldi F, Maroni A, Pandini S. Retentive device for intravesical drug delivery based on water-induced shape memory response of poly(vinyl alcohol): design concept and 4D printing feasibility. Int J Pharm 2019;559:299-311.

[28] Zhang Q, Yan D, Zhang K, Hu G. Pattern Transformation of Heat-Shrinkable Polymer by ThreeDimensional (3D) Printing Technique. Sci Rep 2015;5:24-7. https://doi.org/10.1038/srep08936.

[29] Zhang Q, Zhang K, Hu G. Smart three-dimensional lightweight structure triggered from a thin composite sheet via 3D printing technique. Sci Rep 2016;6:22431. https://doi.org/10.1038/srep22431.

[30] Liu T, Liu L, Zeng C, Liu Y, Leng J. 4D printed anisotropic structures with tailored mechanical behaviors and shape memory effects. Compos Sci Technol 2019:107935. https://doi.org/10.1016/j.compscitech.2019.107935.

[31] Liu Z, Liu H, Duan G, Tan J. Folding deformation modeling and simulation of 4D printed bilayer structures considering the thickness ratio 2019. https://doi.org/10.1177/1081286519877563.

[32] González-henríquez CM, Sarabia-vallejos MA, Rodriguez-hernandez J. Progress in Polymer Science Polymers for additive manufacturing and 4D-printing: Materials, methodologies, and biomedical applications. Prog Polym Sci 2019;94:57-116. https://doi.org/10.1016/j.progpolymsci.2019.03.001.

[33] Zeng S, Gao Y, Feng Y, Zheng H, Qiu H, Tan J. Programming the deformation of a temperature-driven bilayer structure in 4D printing. Smart Mater Struct 2019.

[34] Bodaghi M, Noroozi R, Zolfagharian A, Fotouhi M. 4D Printing Self-Morphing Structures. Materials (Basel) 2019;12.

[35] Zhao Q, Qi HJ, Xie T. Progress in Polymer Science Recent progress in shape memory polymer: New behavior, enabling materials, and mechanistic understanding. Prog Polym Sci 2015;49-50:79-120. https://doi.org/10.1016/j.progpolymsci.2015.04.001. 
[36] Timoshenko BYS. ANALYSIS OF BI-METAL THERMOSTATS. JOSA 1925;11:233-55.

[37] Rayan TP. Statistical Methods for Quality Improvement. John Wiley @ Sons; 1989.

[38] Koeller RC. Applications of Fractional Calculus to the Theory of Viscoelasticity. J Appl Mech 1984;51:299-307.

[39] Fang C, Sun H, Gu J. Application of Fractional Calculus Methods to Viscoelastic Response of amorphous shape memory polymers. J Mechan 2015;31. https://doi.org/10.1017/jmech.2014.98.

[40] Fang Q, Sun H, Gu P. A FRACTIONAL CALCULUS APPROACH TO THE PREDICTION OF FREE RECOVERY BEHAVIORS OF AMORPHOUS SHAPE MEMORY POLYMERS. J Mechan 2016;32:11-7.

[41] Lendlein A, Langer R. Biodegradable, Elastic Shape-Memory Polymers for Potential Biomedical Applications. Science (80- ) 2002;296:1673-6.

[42] Katsourinis S, Kontou E. Fractional viscoelastic models for interconverting linear viscoelastic functions of various polymeric structures. Rheol Acta 2019;58:307-20. https://doi.org/10.1007/s00397-019-01146y.

[43] Yang TC, Wu TL, Hung KC, Chen YL, Wu JH. Mechanical properties and extended creep behavior of bamboo fiber reinforced recycled poly(lactic acid) composites using the timeerature superposition principle. Constr Build Mater 2015;93:558-63. https://doi.org/10.1016/j.conbuildmat.2015.06.038.

[44] Kaw A. Mechanics of Composite Materials. 2nd ed. 2006. https://doi.org/10.1016/C2011-0-05224-9. 


\section{Supplementary Files}

This is a list of supplementary files associated with this preprint. Click to download.

- Appendixl.docx 